\title{
LA CULTURA DE LA LEGALIDAD Y SU COBERTURA EN LA PRENSA NACIONAL MEXICANA*
}

\author{
Citlalli Sánchez Hernández \\ Tecnológico de Monterrey, México
}

http://dx.doi.org/10.5209/rev_NOMA.2013.42354

\begin{abstract}
Resumen.- La Cultura de la Legalidad tiene como premisa la existencia de una sociedad informada de sus leyes y conocedora de las instituciones y organismos que conforman su estructura económica, política y social, ya que en tanto más conozca de sí misma, será participativa e involucrada en su desarrollo. Una parte importante en la socialización de la cultura de la legalidad son los medios de comunicación, pues tienen las herramientas, el alcance y las posibilidades para acercarse a sus públicos e informar desde una nueva perspectiva. El presente trabajo analiza tres periódicos nacionales, Excelsior, La Jornada y El Universal, para identificar la forma en que la prensa aborda los principios de la cultura de la legalidad en su información. Se analizaron los medios de mayor circulación nacional y de fácil acceso a su contenido, de los cuales fueron seleccionadas las noticias sobre violencia, comisión de delitos e inseguridad. El instrumento de evaluación consta de ocho variables principales que operacionalizan los ocho principios de la cultura de la legalidad. La importancia de este proyecto recae en el impacto que pueda tener directamente en los medios noticiosos y contribuir al fomento de la cultura de la legalidad y de una sociedad informada sobre sí misma.
\end{abstract}

Palabras clave.- cultura de la legalidad, prensa, noticias, análisis de contenido

\section{Culture of lawfulness and the coverage in the mexican national press}

Abstract.- The Culture of Lawfulness is based on the premise of the existence a society informed about the laws and institutions that shape its economic, political and social structure. Because this society will be participatory and involved in its own development to the extent in which it is more aware of itself. Mass media plays an important role in the socialization of the culture of lawfulness because they have the tools and possibilities to approach and inform their audiences with a new perspective. In this article, three national newspapers: Excelsior, La Jornada and El Universal, are analyzed to identify how the press deals with the principles of the culture of lawfulness in their information. In these media of major circulation, the news of violence, crimes and insecurity were selected. The content analysis consisted of eight main variables that reflect/represent the eight principles of the culture of lawfulness. The relevance of this project lies in the potential impact it may have on mass media, in order to develop a culture of lawfulness and and a society informed about itself.

"Citlalli Sánchez Hernández. Tecnológico de Monterrey campus Monterrey. Contacto: citla.sanchez@gmail. Doctoranda en Estudios Humanísticos con especialidad en Comunicación y Estudios Culturales, Tecnológico de Monterrey campus Monterrey. Maestra en Ciencias en Comunicación con especialidad en Comunicación Internacional por el Tecnológico de Monterrey campus Monterrey. Licenciada en Ciencias de la Comunicación por la Universidad Autónoma de San Luis Potosí. Asistente de investigación en el Centro de Investigación en Comunicación (CINCO) del Tecnológico de Monterrey desde 2006, donde ha participado en proyectos conjuntos para la Universidad de Texas, la Televisora Estatal de Nuevo León, y el Centro Nacional de Prevención del Delito y Participación Ciudadana. Coordina el monitoreo y análisis de los medios locales y nacionales para el Observatorio de Medios y Cultura de la legalidad (2011-2012), así como la capacitación de codificadores y el diseño de los instrumentos de análisis de contenido. 


\section{Introducción}

La cultura de la legalidad es una propuesta que surge de la necesidad de orientar a la sociedad hacia un estado de derecho, para lo cual se necesita de la intervención directa de ciudadanos involucrados en la vida política de su país, dispuestos a colaborar en la elaboración de políticas públicas (en un nivel ideal) pero sobre todo en el cumplimiento de las normas básicas de convivencia. Se menciona en la visión del National Strategy Information Center (NSIC) -principal promotor de la cultura de la legalidad- que durante mucho tiempo se ha pensado que la democracia electoral es el mejor camino, hay quien piensa que el único, para poder lograr el desarrollo económico, la justicia social y la estabilidad de una sociedad, sin embargo, para esto se requiere de instituciones fortalecidas y comprometidas, que ciudadanos y gobierno sientan el interés y la necesidad de trabajar por un objetivo en común.

Sin embargo, se sabe que ni el delito ni la corrupción han cedido ante las mejoras de nuestro sistema democrático ni del fortalecimiento de las instituciones, ni por la mejora en la participación de la sociedad civil -dice Salazar (2006) que la democracia por sí misma no garantiza la legalidad, y la legalidad por sí misma no garantiza la justicia- de modo que la cultura de la legalidad surge como esa propuesta que sirva como unificador entre gobierno y sociedad a favor del estado de derecho y la prevención del delito y la corrupción. Como señala la visión de NSIC, "tienen (gobierno y sociedad civil) que compartir la expectativa de que las leyes deben ser justas y se aplican a todos, independientemente de la situación socioeconómica, y que cada individuo tiene un papel en la creación y supervisión de la aplicación de las leyes". Eso es lo que pretende la cultura de la legalidad.

Ahora bien ¿cómo se relaciona la cultura de la legalidad con el quehacer de los medios de comunicación? ¿cuál es el papel de la información noticiosa? Una de las herramientas de la cultura de la legalidad son los medios de comunicación, y en particular los medios impresos fueron parte fundamental en la reestructuración social de Palermo, la ciudad donde inicia la propuesta de la cultura de la legalidad: gracias al apoyo del Giornale de Sicilia, las personas tuvieron acceso a información sobre sus instituciones de seguridad, el seguimiento a los casos de detenciones de los capos de la mafia, la explicación de los delitos que se cometían y los castigos correspondientes a ellos, los procedimientos para la denuncia de actos de corrupción o hechos delictivos. Y por otro lado, se dio la posibilidad de colaborar con ideas para el mejoramiento de las acciones y programas de prevención del delito que incidían en las políticas públicas sobre seguridad.

El monitoreo que realiza el Observatorio de Medios y Cultura de la Legalidad, y del que este trabajo toma una parte para analizar la prensa de circulación nacional, 
tiene la intención de colaborar tanto con los medios como con las instituciones de gobierno: al promover la cultura de la legalidad como parte importante a cubrir en la información noticiosa, se cree que las audiencias pueden empezar a conocer más sobre procedimientos de denuncia y acciones para la prevención del delito y la corrupción; se puede incidir en las políticas editoriales para que la información noticiosa se convierta en esa herramienta que tanta falta hace --informar más allá de la descripción de los hechos, aportando un poco más para que la audiencia pueda tomar decisiones--; y por último, para que desde el gobierno se considere la cultura de la legalidad como una alternativa unificadora, como se planteaba al inicio, que le permita a las instituciones acercarse a la sociedad civil y trabajar en conjunto.

La presente investigación tiene como objetivo realizar un análisis de la forma en que tres diarios nacionales -La Jornada, Excelsior y El Universal- abordan los ocho principios de la cultura de la legalidad en sus noticias relacionadas con violencia, hechos delictivos e inseguridad. Se analizaron cinco semanas naturales correspondientes a los meses de julio a noviembre del 2011, y se realizó un análisis de contenido en el que cada nota fue revisada de modo particular. La importancia de este trabajo recae justamente en la necesidad actual de unir los esfuerzos para que todas las instituciones -incluyendo a los medios de comunicación- se integren a participar de la propuesta de la cultura de la legalidad.

\section{Medios y violencia: la cultura de la legalidad como punto convergente}

La seguridad pública es un asunto que por su naturaleza social y al ser parte necesaria para la adecuada convivencia, se encuentra presente en las agendas temáticas tanto de los gobiernos como de los medios de comunicación. El Informe sobre la seguridad ciudadana y derechos humanos (2010), realizado por la Organización de Estados Americanos señala que la seguridad es una de las grandes demandas de la sociedad, debido principalmente a los niveles de violencia y criminalidad que van en aumento, y que a pesar de ser un derecho regulado por sin fin de políticas públicas "se ve amenazada cuando el Estado no cumple con su función de brindar protección ante el crimen y la violencia social, lo cual interrumpe la relación básica entre gobernantes y gobernados" (p. 1).

Esta relación entre violencia-ciudadanos-Estado no es tan simple de explicar, pues las culpas y obligaciones no recaen solamente de un lado, es decir, los ciudadanos reclaman la ineficacia de las autoridades en materia de seguridad, pero por otro lado se reconoce que la ciudadanía no posee las herramientas y conocimientos adecuados para dejar de reproducir prácticas y patrones basados en la violencia. Explica Garzón (2010), que más allá de la confrontación entre incompetencia del Estado contra la falta de participación ciudadana, existe una relación entre violencia y legalidad, que no es directamente proporcional, y que por el contrario hay una falta de comprensión de los factores que integran ambos conceptos,

La ilegalidad no siempre ha equivalido a dosis altas de violencia, ni la baja en los indicadores de violencia ha implicado que se haya reducido la ilegalidad (o que 
haya más estatalidad). En México, la tendencia a la baja de la tasa de homicidios (dolosos) en el período de 2001 a 2007 no necesariamente se encuentra relacionada con una contracción de la ilegalidad o una menor actividad de los carteles (para. 1).

Bajo esta perspectiva, tenemos entonces la situación de las acciones violentas, ejercidas ya sea por los grupos del crimen organizado, por los ciudadanos, o incluso por las mismas autoridades, y por otro lado la efectividad de las políticas públicas y de las acciones ciudadanas que intentan mantener el Estado de Derecho, es decir, lograr la convivencia bajo el marco de la legalidad.

Para Garzón (2010) existen cuatro formas posibles en las que se puede presentar la interacción de violencia-legalidad, y que resulta necesario revisar para lograr comprender más a fondo cómo es que tanto la sociedad civil como las instituciones (aquí se incluye a los medios de comunicación) colaboran en estos procesos de seguridad ciudadana: (a) Ciudadanos violentos, un escenario en el que la violencia es atribuida a las personas en general, como la forma inmediata para la resolución de sus conflictos (la llamada cultura de la violencia), y existe poco ejercicio de la legalidad por parte del Estado; (b) Estado fuerte, en donde las autoridades logran reducir los índices de actividad ilegal, y se generan también pocas acciones tendientes a la violencia; (c) Todos contra todos, donde la incidencia es de altos niveles de violencia combinados por altos niveles de ilegalidad; (d) Monopolio ilegal, el último escenario en donde a pesar de los elevados índices de actividades ilegales, la violencia no se hace presente en el mismo nivel.

De acuerdo con esta tesis, tenemos que violencia y delincuencia no es lo mismo, que atiende a factores diversos, pero que ambas cosas tienen su punto de encuentro en la participación conjunta de instituciones y ciudadanos, ya sea para elevar los índices o para intentar disminuirlos. Bajo estas premisas ¿en qué escenario estaría posicionado México? ¿cómo es que los medios de comunicación tendrían cabida en este escenario? Para Garzón (2010), México está situado en el tercero, todos contra todos: los distintos grupos del crimen organizado compitiendo entre sí y contra el gobierno, y las autoridades a su vez, buscando imponer acciones orientadas a terminar con la ilegalidad que estos grupos generan. $Y$ en medio, el ciudadano.

Tenemos entonces que el problema de la inseguridad y de la violencia en nuestro país tiene que ver en gran medida con la ineficiencia de las autoridades y las instituciones, es notable que no hay una impartición de justicia clara ni eficiente, pero también es necesario considerar que la otra parte de responsabilidad recae en los ciudadanos. En la medida en que todos participen en exigir a las autoridades y estén dispuestos a colaborar con ella, existen más posibilidades de que se logre establecer un estado de derecho.

Apunta Salazar (2006) que un estado de derecho tiene su base en el reconocimiento de la dignidad, y bajo esta premisa es que se modelan e instauran las instituciones, cuyo principal objetivo será garantizarla. El miedo no es la base de un estado de derecho, es la convicción de que se hace lo adecuado. Para 
Godson (2000) el estado de derecho implica que todos deben ser tratados por igual, y es este -y no necesariamente la elección democrática de la mayoría- lo que brinda protección a todos los miembros de una sociedad. Menciona Salazar (2006) que la ley per se, es decir, la legalidad, no es una garantía de buena gobernanza porque la actuación del poder legal no necesariamente está orientado a la protección de los derechos individuales.

No resulta suficiente contar con un aparato institucional fortalecido, como indica Godson (2000), la prevención y reducción del delito debe abordarse desde un enfoque regulatorio pero necesita ser reforzado por una cultura simpatizante que apoye el cambio en los valores, necesita ser aceptado por una sociedad convencida y dispuesta. Señala Orlando (2003) respecto a las acciones y estrategias que se tuvieron que implementar para lograr un cambio tan radical en la ciudad de Palermo, que "...nuestra lucha mostraba que los tribunales son solamente la línea de frente de la campaña contra la violencia y el desacato a la ley. El otro frente es la cultura" (p. 14). Que quien infrinja la ley reciba la sanción correspondiente, dice Godson (2000), pero también debe ser señalado por otros sectores de la sociedad, distintos al poder judicial. Por ejemplo, de acuerdo con el Índice Nacional de Corrupción y Buen Gobierno 2011 (INCBG 2011), en 2007 se identificaron 197 millones de actos de corrupción en donde están implicadas desde autoridades locales hasta federales, así como servicios particulares; para 2010 esta cifra aumentó a 200 millones. Tan solo en 2010 se estima que 32 mil millones de pesos fueron destinados al pago de "mordidas", es decir, un $14 \%$ del ingreso de una familia promedio. Sin duda que se trata de conductas abiertamente criticadas, pero que en muchas ocasiones se carece de la convicción suficiente para evitar involucrarse con ellas y eso es justo, lo que propone la cultura de la legalidad: fomentar el arraigo desde la cultura, por el respeto a las leyes y el apego a estas.

La cultura de la legalidad es entonces cuando "la cultura, ethos y pensamiento dominante de una sociedad simpatizan con la observancia de la ley" (Godson, 2000 , p. 2), y no surge forma espontánea, se trata de provocar en la sociedad la convicción por el apego a la norma, que las conozcan, las acepten y logren un arraigo en la forma de vida, que sean criterios sobre cómo actuar (Salazar, 2006). Se trata de que la persona promedio crea en las normas legales como parte necesaria para la impartición de justicia, es por eso que se habla de una cultura, porque requiere de procesos de adaptación, de empatía. La ley sin una cultura que simpatice con ella, no tiene la misma eficiencia, "la cultura necesita la ejecución de la ley, pero los ejecutores necesitan la cultura" (Godson, 2000, p. 3). Para Laveaga (2006), la cultura de la legalidad es

El conocimiento que un pueblo tiene de su derecho, así como los esfuerzos que hacen los grupos y facciones - principalmente el gobierno- para difundir o no difundir tal conocimiento, las variables del proceso mediante el que un pueblo acata las normas que lo rigen, los efectos concretos que este ejercicio concreto tiene en la sociedad civil y los límites a que se circunscribe" (p. 20).

Los ocho principios que propone la cultura de la legalidad son los siguientes: (1) La sociedad conoce la mayoría de las normas y leyes; (2) La mayoría tienen la 
voluntad de respetar las leyes; (3) Existe una rama estructurada y órganos de control. La sociedad está controlada y estructurada legalmente; (4) Existe una sanción o castigo para la persona o personas que cometan una violación de ley; (5) Existe un proceso de defensa de acusados. Al ciudadano que supuestamente incurra en una violación de ley se le debe hacer valer un proceso para su defensa; (6) Existe un sistema de apoyo a víctimas. Se podrá otorgar ayuda a las personas que hayan sido afectadas por una agresión penada por la ley; (7) Se condena a la delincuencia y corrupción; (8) Existe un sistema para cambiar las leyes cuando sea pertinente.

¿Cuál es entonces el papel de los medios de comunicación en este proceso de socialización de la cultura de la legalidad? Se considera que existen varios elementos e instituciones que apoyan su reforzamiento: (a) Educación cívica y escolar, (b) Centros de autoridad moral, (c) Los medios y la cultura popular, (d) Las corporaciones policiacas (Godson, 2000). El papel de los medios sería el de la vigilancia de los funcionarios, por ejemplo, que cumplan adecuadamente y de acuerdo a la ley con sus obligaciones y si no lo hacen, exponerlo ante la sociedad. Por otro lado, también les corresponde el fomento y la promoción de la participación en las acciones y estrategias de la cultura de la legalidad dando voz a los activistas y al público en general como el caso del Giornale de Sicilia, que fue una herramienta primordial en el combate a la delincuencia.

Aunado a lo anterior, es necesario reconocer el impacto de los medios masivos en la conformación de percepciones, ideas y representaciones hacia cualquier ámbito de la vida social, que existe una presencia notable de los medios en la vida cotidiana de niños y jóvenes que forma parte de sus procesos de socialización. Aunque en menor medida que la televisión y la radio, los diarios impresos siguen siendo una fuente recurrente para una parte de la población, son una fuente de fácil acceso por la cual se puede conocer lo que acontece cerca o lejos de la localidad, cuando los hechos violentos parecen ser el tema constante en la agenda mediática surge el cuestionamiento sobre cuál tendría que ser la cobertura de los medios respecto a temas de violencia y narcotráfico, sin dejar de informar a la sociedad, pero tomando en cuenta los principios de la cultura de la legalidad.

\section{Metodología}

El presente análisis fue realizado con los datos obtenidos del monitoreo que realizó el Observatorio de Medios y Cultura de la Legalidad durante los meses de julio a noviembre de 2011 , de cada mes fue seleccionada la segunda semana natural, de modo que los datos corresponden a cinco semanas del año. Fueron analizadas las ediciones impresas de La Jornada, Excelsior y El Universal, en las que se revisaron las secciones Nacional, Local, Policiaca, Seguridad, Internacional (en cada diario pueden variar los nombres de las secciones).

La unidad de análisis es la nota informativa que se refiere directa y explícitamente a conductas delictivas, inseguridad, narcotráfico, corrupción, abuso de poder, etc., o a propuestas, reacciones o críticas a dichos temas. Se incluyen las notas publicadas en las secciones internacionales que cumplan con los criterios 
anteriores y que se refieran o involucren directamente a México o a mexicanos, ya sea en territorio nacional o extranjero. Se tomó la decisión de excluir editoriales, columnas de periodistas a título personal, artículos de opinión, crítica o infoentretenimiento. En total se analizaron 1040 notas que cumplen con la definición establecida $(\mathrm{N}=1040)$, de las cuales La Jornada tiene $364(\mathrm{n}=364)$, Excelsior reporta 377 ( $n=377)$, y El Universal 376 ( $n=376)$.

El instrumento utilizado para el análisis de contenido consta de 43 variables, en la primera parte se operacionalizan los ocho principios de la cultura de la legalidad sugeridos por Godson; el resto de las variables miden aspectos como la participación ciudadana en el mensaje, los tipos de fuente, tratamiento de la información, su espectacularización, y uso de recursos audiovisuales. Para esta investigación se tomaron en cuenta únicamente las variables relacionadas con la representación de los principios de la cultura de la legalidad. ${ }^{\dagger}$

\section{Hallazgos en los noticieros nacionales}

La cultura de la legalidad promueve ocho principios orientados a fomentar el estado de derecho, prevenir y reducir el delito, y generar las condiciones culturales para que la sociedad adquiera criterios sobre su actuación cotidiana bajo la observancia de la ley. Con la intención de conocer en qué medida se representan estos principios, se analizó el contenido de las notas de tres diarios de circulación nacional cuya información abordaba temáticas como la violencia, la inseguridad, el narcotráfico y los hechos delictivos.

Tabla 1. ¿En qué medida se cumplen los principios de la cultura de la legalidad en los periódicos nacionales mexicanos?

\begin{tabular}{|l|l|l|l|l|}
\hline \multirow{2}{*}{$\begin{array}{l}\text { Principio de la Cultura } \\
\text { de la Legalidad }\end{array}$} & Variable & \multicolumn{2}{|c|}{ Cumple Suficiente } \\
& *(julio a noviembre de 2011) \\
\cline { 2 - 5 } & La Jornada & Excelsior & El Universal \\
\hline $\begin{array}{l}\text { 1. La sociedad conoce } \\
\text { la mayoría de las } \\
\text { normas y leyes. }\end{array}$ & $\begin{array}{l}\text { 1. El mensaje } \\
\text { menciona las normas } \\
\text { o leyes violadas o que } \\
\text { no se aplicaron. }\end{array}$ & $55 \%$ & $61 \%$ & $59 \%$ \\
\hline $\begin{array}{l}\text { 2. La mayoría tienen } \\
\text { la voluntad de } \\
\text { respetar las leyes. }\end{array}$ & $\begin{array}{l}\text { 2. El mensaje enfatiza } \\
\text { la necesidad y } \\
\text { conveniencia de }\end{array}$ & $23 \%$ & $48 \%$ & $48 \%$ \\
\hline
\end{tabular}

\footnotetext{
${ }^{\dagger}$ Para convertir cada variable en un principio, se operacionalizó de la siguiente forma: Principio: La sociedad conoce la mayoría de las normas y leyes. Las personas de una sociedad conocen las partes esenciales de las leyes más importantes del documento oficial que rige a estas. Variable: El mensaje menciona las normas o leyes violadas o que no se aplicaron (Se debe considerar si por el tipo de información, la nota debe incluir lo mencionado en la variable. Si no es así, entonces deberá elegirse la opción de no aplica.)

Indicadores: 1. No; 2. Insuficiente; 3. Suficiente; 4. No aplica.
} 


\begin{tabular}{|c|c|c|c|c|}
\hline & $\begin{array}{l}\text { respetar las leyes } \\
\text { para solucionar } \\
\text { problemas. }\end{array}$ & & & \\
\hline $\begin{array}{l}\text { 3. Existe una rama } \\
\text { estructurada y } \\
\text { órganos de control. La } \\
\text { sociedad está } \\
\text { controlada y } \\
\text { estructurada } \\
\text { legalmente. }\end{array}$ & $\begin{array}{l}\text { 3. El mensaje destaca } \\
\text { o promueve la } \\
\text { necesidad de } \\
\text { consensos, acuerdos } \\
\text { o visión de estado } \\
\text { entre distintos actores } \\
\text { políticos y/o } \\
\text { ciudadanos. }\end{array}$ & $47 \%$ & $60 \%$ & $71 \%$ \\
\hline $\begin{array}{l}\text { 4. Existe una sanción } \\
\text { o castigo para la } \\
\text { persona o personas } \\
\text { que cometan una } \\
\text { violación de ley. }\end{array}$ & $\begin{array}{l}\text { 4. Destaca o } \\
\text { promueve la } \\
\text { necesidad de aplicar } \\
\text { sanciones y/o atacar y } \\
\text { disminuir la impunidad } \\
\text { como solución } \\
\text { estructural a delitos, } \\
\text { inseguridad y } \\
\text { corrupción. }\end{array}$ & $25 \%$ & $46 \%$ & $54 \%$ \\
\hline $\begin{array}{l}\text { 5. Existe un proceso } \\
\text { de defensa de } \\
\text { acusados. Al } \\
\text { ciudadano que } \\
\text { supuestamente } \\
\text { incurra en una } \\
\text { violación de ley se le } \\
\text { debe hacer valer un } \\
\text { proceso para su } \\
\text { defensa. }\end{array}$ & $\begin{array}{l}\text { 5. Destaca y } \\
\text { promueve la } \\
\text { necesidad de respetar } \\
\text { el derecho de los } \\
\text { acusados a la } \\
\text { presunción de } \\
\text { inocencia y de su } \\
\text { derecho a procesos } \\
\text { de defensa y juicio. }\end{array}$ & $16 \%$ & $38 \%$ & $50 \%$ \\
\hline $\begin{array}{l}\text { 6. Existe un sistema } \\
\text { de apoyo a víctimas. } \\
\text { Se podrá otorgar } \\
\text { ayuda a las personas } \\
\text { que hayan sido } \\
\text { afectadas por una } \\
\text { agresión penada por } \\
\text { la ley. }\end{array}$ & $\begin{array}{l}\text { 6. Destaca y } \\
\text { promueve el apoyo } \\
\text { legal, material, } \\
\text { psicológico y/o de } \\
\text { seguridad de las } \\
\text { víctimas de } \\
\text { agresiones penadas } \\
\text { por la ley. }\end{array}$ & $15 \%$ & $28 \%$ & $37 \%$ \\
\hline $\begin{array}{l}\text { 7. Se condena a la } \\
\text { delincuencia y } \\
\text { corrupción. }\end{array}$ & $\begin{array}{l}\text { 7. Denuncia y/o } \\
\text { condena la } \\
\text { delincuencia y la } \\
\text { corrupción. }\end{array}$ & $40 \%$ & $55 \%$ & $63 \%$ \\
\hline
\end{tabular}




\begin{tabular}{|c|c|c|c|c|}
\hline $\begin{array}{l}\text { 8. Existe un sistema } \\
\text { para cambiar las leyes } \\
\text { cuando sea } \\
\text { pertinente. }\end{array}$ & \begin{tabular}{|l|} 
8. Menciona la \\
necesidad de cambiar \\
leyes o normas que \\
impiden las \\
transformaciones o \\
estrategias necesarias \\
y adecuadas.
\end{tabular} & $41 \%$ & $52 \%$ & $49 \%$ \\
\hline
\end{tabular}

Fuente: Elaboración propia

El análisis general de la cobertura de los principios de cultura de la legalidad en la prensa nacional, arroja hallazgos interesantes, pues al menos tres principios fueron abordados de forma Suficiente en el $50 \%$ o más de las notas totales de la muestra: P1. "Mención de las leyes...", P3. "Necesidad de consensos...", P7. "Denuncia de la delincuencia...", lo que resulta importante porque indica que en al menos la mitad de las noticias se está presentando de forma clara y explícita cuáles son los delitos que se están cometiendo o las leyes que no se han respetado lo que permite que los lectores tengan la información más completa; además el medio está aprovechando sus recursos para manifestar su inconformidad ante los hechos delictivos y condenando los actos de corrupción, que es una forma de guiar a los lectores al señalar sin miedos aquello que daña a la sociedad, y hace hincapié en que uno de los elementos primordiales en la prevención del delito recae en el diálogo de los actores institucionales y de la sociedad civil, la toma de responsabilidades y la necesidad de acuerdos entre las partes.

Por otro lado, existen tres variables que calificaron como Suficiente entre el 42 y el $48 \%$ de las noticias: P2. "Énfasis en el respeto a las leyes...", P4. "Necesidad de aplicar sanciones...", P8. "Cambio de leyes como estrategia...", si bien no representa la mayoría de las notas, sí es un avance el que la información de la prensa no sólo mencione los delitos que se cometen o las leyes que se infringen sino que también se mencione cuál es la penalidad correspondiente para cada infracción, pues de este modo se destaca la importancia de prevenir el delito desde el respeto a las normas. Aunado a esto, este porcentaje de noticias que aborda de forma clara la posibilidad de que las leyes puedan ser modificadas 0 cambiadas, permite que los lectores conciban a la legislación como algo que puede ser flexible y se adapte las necesidades de la sociedad, y que puede ser una estrategia efectiva para la impartición de justicia.

A pesar de que la mayor parte de los principios de la cultura de la legalidad parecen estar presentes de algún modo en los contenidos noticiosos, hay dos que no son considerados en las noticias pero que por su naturaleza deberían ser parte importante de la información de una nota sobre hechos delictivos, corrupción o violencia. El primer caso es el del P6. "Destaca el apoyo a víctimas de delitos...", que reportó en el análisis general un balance de $52 \%$ de las notas en donde, teniendo la posibilidad de abordar o promover este principio, no lo hicieron. El otro principio que reporta una situación atípica, P5. "Destaca la necesidad de respetar el derecho de la presunción de inocencia...", pues los tres indicadores -No, Insuficiente y Suficiente- comparten el $30 \%$ de la notas. Si bien, ningún principio 
es más importante, los temas de la atención a víctimas y el respeto a un juicio justo se han discutido en últimas fechas en los medios mexicanos, sobre todo a raíz del incremento en los hechos violentos.

\section{Sobre las leyes: ¿se conocen? ¿se respetan? ¿se sanciona?}

Parte de los problemas de corrupción y delincuencia tienen su origen en el desconocimiento de las leyes, normas, derechos y obligaciones, la desinformación sobre aquello que es o no es un delito -más allá de la comprensión ética o moral sobre el bien y el mal- conlleva la posibilidad de cometer faltas que ponen en peligro a la sociedad: desde una infracción de tránsito hasta el soborno a un funcionario público. Cuando un medio reporta la información relacionada con hechos delictivos, tiene la obligación de presentar cuál es la ley que se infringe, de modo que los lectores puedan tener conocimiento de esto. Si bien la educación cívica se recibe en la institución escolar y familiar, los medios tiene la posibilidad de impactar un poco más en este aspecto. En la variable 1 -El mensaje menciona las normas o leyes violadas o que no se aplicaron- no sólo se observa que la ley sea mencionada, sino que cuando se hable de delitos específicos, sean nombrados de la forma correcta, sin eufemismos y lo más apegado a la forma legal que se describe en el Código Penal Federal. Los resultados de este primer principio de la cultura de la legalidad nos indican que los tres periódicos analizados mencionan de forma explícita y clara en el cuerpo de las notas, las leyes o normal que fueron violadas o no se aplicaron. Todos los periódicos reportan una cobertura Suficiente en más del $50 \%$ de sus notas: La Jornada presenta un 55\% $(n=286)$, Excelsior un 61\% $(n=314)$ y El Universal un $59 \%$ $(n=300)$.

Ahora bien, no sólo se trata de conocer las leyes que nos rigen o las instituciones de las que forma parte nuestra estructura política y social. La cultura de la legalidad pretende que, una vez que se conocen las normas, logren comprenderse en el marco de su utilidad, de la forma en que su cumplimiento beneficia a la sociedad y permite el estado de derecho. Es decir, la voluntad de respetar las leyes tendría que surgir de la comprensión de las mismas, y que no sea el respeto únicamente por el castigo que conlleva el no acatar. La responsabilidad del medio recae en destacar en su información el cómo esta voluntad de cumplir puede ser el camino hacia la prevención del delito. En este sentido, los periódicos nacionales presentan una marcada disparidad en la variable 2 -El mensaje enfatiza la necesidad y conveniencia de respetar las leyes para solucionar problemas-, pues mientras Excelsior $(n=258)$ y El Universal $(n=301)$ mencionó de forma Suficiente este principio en un $48 \%$ de las notas, en La Jornada $(n=229)$ la mayoría de las notas -54\%- no se cubrió esta variable.

Para que la ciudadanía pueda confiar en las instituciones tiene que conocerlas, y saber que entre ellas existe una voluntad de colaboración y de interactuar adecuadamente cuando sea necesario. Esta comunicación es necesaria entre instituciones, actos, políticos, ciudadanos, porque la disposición al diálogo es uno de los primeros caminos para poder saber lo que cada sector de la sociedad necesita. Este principio de la cultura de la legalidad tiene su base precisamente en la necesidad de dialogar, de establecer acuerdos como un punto relevante para la 
prevención del delito, y el medio de comunicación tiene la posibilidad de participar desde dos posturas: como un mediador, o como parte de estos actores públicos que intervienen en el debate de la problemática pública en cuestiones de seguridad ciudadana. En la variable 3 -El mensaje destaca o promueve la necesidad de consensos, acuerdos o visión de estado entre distintos actores políticos y/o ciudadanos- resulta interesante observar que los tres diarios analizados reportaron como Suficiente la cobertura de este principio en la mayoría de sus notas: en La Jornada $(n=145)$ se registró un $47 \%$, en Excelsior $(n=215)$ un $60 \%$ y en El Universal $(n=276)$ un $71 \%$, resultando el periódico con el porcentaje más alto en la cobertura de esta variable.

Conocer las leyes no es suficiente para la cultura de la legalidad. Más allá de conocer los reglamentos y normas que rigen nuestra vida en sociedad, resulta importante saber que hay un órgano encargado de vigilar que estas se cumplan y que en caso de ser infringidas, se aplicará un castigo. Saber que se cuenta con una legislación adecuada es importante para la seguridad de la ciudadanía, y de acuerdo a los resultados vistos hasta el momento los periódicos nacionales lo han mencionado en buena parte de sus notas, sin embargo también es necesario saber que en caso de que estas leyes no se cumplan existen sanciones que serán aplicadas. En Palermo, el diario local tenía por política mencionar los delitos cometidos, pero también hacer énfasis en la pena correspondiente, de modo que los lectores tenían claro que nadie que infringiera la ley quedaría sin castigo. En esta variable 4 -Destaca o promueve la necesidad de aplicar sanciones y/o atacar y disminuir la impunidad como solución estructural a delitos, inseguridad y corrupción- los periódicos nacionales presentan notables diferencias, pues nuevamente La Jornada $(n=251)$ reportó el indicador de Suficiente como el más bajo, pues sólo un $25 \%$ de sus notas destacó este principio e incluso la mayoría de sus notas $(46 \%)$ no lo mencionaron. En el caso de Excelsior $(n=215)$ y $\mathrm{El}$ Universal $(n=276)$ ambos diarios mencionaron de forma Suficiente la cuestión de las sanciones para la prevención del delito, con un 46 y 54\% respectivamente.

\section{Sobre la presunción de inocencia y la defensa de las víctimas del delito}

La cuestión del tratamiento a los acusados de algún delito ha sido tema de controversia, debido a los casos de violación de los derechos humanos a los que son sometidos quienes resultan sospechosos. Este quinto principio de la legalidad aboga por el derecho a la defensa de los involucrados y que se respete la presunción de inocencia hasta que las evidencias ofrecidas en un juicio llevado bajo el debido proceso demuestren que es culpable. La forma en que los medios impactan en estos casos es notable, pues en muchas ocasiones los mismos reporteros realizan juicios tempranos sobre la situación legal de un detenido, sin que este haya pasado por un juicio, son presentados ante las cámaras y se mencionan los datos personales. Si bien la cultura de la legalidad pretende que la sociedad se informe sobre asuntos de seguridad, es importante que se respeten las instancias por las cuales se llevan a cabo los juicios, sin poner en peligro a los detenidos y a sus familias. 
A pesar del revuelo que ha causado el debate sobre la presunción de inocencia, sobre todo a raíz del documental mexicano "Presunto culpable" que narra la situación de un hombre que se ve involucrado en hechos delictivos y es condenado injustamente, no todos los medios impresos nacionales muestran interés en la cobertura de esta variable -Destaca y promueve la necesidad de respetar el derecho de los acusados a la presunción de inocencia y de su derecho a procesos de defensa y juicio-, pues aunque El Universal $(n=117)$ registró un $50 \%$ de sus notas como Suficiente, y Excelsior ( $n=144$ ) reportó un $41 \%$ de su información como Insuficiente, es decir, aunque en sus notas trata a los sospechosos como "presuntos", el cuerpo de la nota deja entrever que hay una acusación preestablecida por el reportero; en el caso de La Jornada ( $n=151)$ un $51 \%$ de las notas no respetan este principio.

Un tema que es necesario discutir, no sólo en el marco de la cultura de la legalidad, sino en general respecto a las políticas públicas de seguridad ciudadana, es el del seguimiento a las víctimas, ya sea quienes en carne propia fueron objeto de un hecho delictivo, como también de las familias afectadas y de los testigos presenciales. En la información periodística es importante señalas los acontecimientos y sus protagonistas, y en los casos en que el medio realiza un seguimiento de los acusados, no lo hace respecto a quienes se vieron involucrados y resultaron afectados de algún modo. Un sistema legislativo justo es aquel que tiene la capacidad de castigar a quien infringe la ley, pero también de resarcir a quienes se haya causado el daño, y es aquí donde los medios de comunicación tienen la posibilidad de dar visibilidad a estas personas, pero no desde la espectacularización de la noticia, sino desde su posición para presionar a las autoridades en los procesos de interposición de demandas, exigencia de indemnizaciones, apoyo material o psicológico y de este modo informar al resto de la ciudadanía sobre cómo es posible hacerlo.

Como se revisaba en el análisis general, este principio -Destaca y promueve el apoyo legal, material, psicológico y/o de seguridad de las víctimas de agresiones penadas por la ley- es el que se reportó en mayor medida como no mencionado. En el caso de El Universal $(n=185)$ del total de notas se abordó de forma Suficiente en un $37 \%$, que es el porcentaje más alto ente los demás indicadores y también frente al resto de los periódicos. En Excelsior $(n=161)$ un $50 \%$ de las notas fueron calificadas como No, y en La Jornada $(n=186)$ este mismo indicador llegó a un $72 \%$, es decir, las víctimas prácticamente no son parte de la información que reportan ni mucho menos hay un seguimiento a estas.

\section{Sobre la corrupción y la flexibilidad de la ley}

Poco a poco y a fuerza de ser objeto de múltiples atropellos, la sociedad mexicana va levantando la voz, comenzando a organizarse y aprendiendo la importancia de señalar, de hacer denuncia de los actos delictivos. Una muestra de esto son las organizaciones civiles que han ido creándose en México, muchas enfocadas a la atención a víctimas, otras más a la prevención del delito, algunas más sobre la promoción de la cultura de la legalidad -como el caso del Observatorio de Medios 
y Cultura de la Legalidad-. La denuncia es importante para la seguridad ciudadana, porque permite poner de manifiesto las arbitrariedades de las instituciones pero también la mala actuación de algunos miembros de la sociedad. La propuesta de la cultura de la legalidad es que los medios se involucren también en la acción de denunciar, y dado que cuentan con los recursos para hacerlo, involucren y orienten de esta forma a la ciudadanía. Los diarios nacionales que se analizaron arrojan resultados interesantes, pues respecto a la variable 7 -Denuncia y/o condena la delincuencia y la corrupción- todos reportaron la mayoría de noticias en donde este principio se aborda de forma Suficiente: La Jornada ( $\mathrm{n}=$ $305)$ con un $50 \%$, Excelsior $(n=298)$ con $55 \%$ y El Universal $(n=348)$ con $63 \%$.

Cuando se habla de leyes, se piensa en las constituciones y legislaciones muchas veces anacrónicas, que a pesar de la necesidad de ser renovadas existe una renuencia a hacerlo aunque muchas veces alguna ley esté por encima de los derechos y garantías individuales. Este principio de la cultura de la legalidad plantea un sistema de leyes eficiente pero flexible: la sociedad cambia, las costumbres se van modificando, por lo tanto es justo tener la seguridad de que existe la posibilidad de que las leyes también puedan adaptarse, sobre todo a favor de un estado de derecho interesado en proteger la dignidad y proporcionar justicia. Existen delitos que han requerido de debates para modificar ya sea las penas que se van a aplicar, su tipificación en los códigos penales -para inclusión o para modificación-, o los procesos con los cuales se realizan los juicios. El seguimiento que los medios noticiosos le dan a estos acontecimientos, porque los lectores pueden estar informados de la forma en que opera el sistema legislativo y judicial, y tiene la posibilidad de formarse una opinión al respecto. En el caso de los periódicos nacionales, se encontró nuevamente que en la mayoría de las notas hay un alto porcentaje de incidencia en el abordaje de la variable 8 -Menciona la necesidad de cambiar leyes o normas que impiden las transformaciones 0 estrategias necesarias y adecuadas-, La Jornada ( $n=105)$ reportó que un $41 \%$ de de sus notas califica como Suficiente en esta variable, mientras que Excelsior ( $\mathrm{n}=$ $125)$ alcanzó un $52 \%$ y El Universal $(n=187)$ un $63 \%$.

\section{Conclusiones}

La presente investigación tuvo por objeto analizar la forma en que las noticias que se transmiten en los diarios impresos nacionales - La Jornada, Excelsior y El Universal- abordan y consideran los principios de la cultura de la legalidad en las noticias relacionadas con violencia, inseguridad y hechos delictivos. Partiendo de la importancia de los medios informativos en la formación de criterios respecto a la política, y en particular las temáticas relacionadas con la seguridad, debe reconocerse la necesidad de que la propuesta de la cultura de la legalidad empiece a ser considerada en las políticas editoriales de los medios.

Los datos que se analizaron fueron obtenidos del monitoreo que realiza el Observatorio de Medios y Cultura de la Legalidad, que desde el 2010 se ha dedicado generar indicadores sobre la forma en que los medios consideran a los principios de la cultura de la legalidad en sus contenidos y en cómo el público - 
particularmente el regiomontano- recibe esta información. Las reflexiones que surjan alrededor de esto tienen como meta contribuir al fomento de una cultura de la legalidad tanto en los ciudadanos interesados como en los medios de comunicación.

El convencer a los medios de comunicación para que participen de esta iniciativa implica un gran reto, pues seguirán enfocándose en resaltar aspecto de la espectacularización de la nota mientras las rutinas periodísticas no se modifiquen, las autoridades no brinden seguridad a los periodistas y en tanto no se genere una verdadera conciencia sobre la importancia y ventaja que tiene vivir en un estado de derecho. Los medios tienen la consigna de colaborar activamente para poder logar el cambio, como ha sucedido en otras ciudades del mundo.

Si bien no hay un principios más importante que otro, hay algunos que podrían pensarse como básicos, dada la situación social de nuestro país, como el caso de la presunción de inocencia, y el seguimiento a las víctimas de hechos delictivos. Se esperaría que, dada la polémica que han causado los casos de personas aprehendidas y juzgadas sin respeto a sus derechos, los medios tendrían más precaución al momento de abordar estos temas, sin embargo sigue siendo un tema que se maneja con descuido. Al igual que la cobertura de las víctimas, que siguen siendo las últimas en la lista de prioridades tanto para los medios como para las instituciones. La noticia se enfoca tanto en describir a quienes cometen los crímenes, que se deja de lado a quienes sufren las consecuencias: la primera plana puede dedicársele por completo a la aprehensión de un capo del narcotráfico, pero pocas veces serán las víctimas de un atentado -como el caso del Casino Royale en Monterrey- a quienes se les de tanta importancia.

Dice Salazar (2006) que mientras en el país siga habiendo desigualdades graves en todos los ámbitos de la vida -de los social a lo económico- la sociedad seguirá siendo el caldo de cultivo para la violencia en general -de lo civil a lo político, de lo público a lo privado-, y añade "la cultura de la legalidad que imagino (...) tiene más que ver con la solidaridad, la corresponsabilidad, el sentido de lo público, la tolerancia y el contacto interpersonal" (p. 50). De modo que cada contribución que los medios de comunicación pueden hacer a este ideal de cultura de la legalidad, será de vital importancia para poder comenzar a desarraigar la cultura de la corrupción, la impunidad y la desconfianza, y comenzar a construir una mejor forma de convivencia. Un ciudadano informado es un individuo con poder, que sabrá exigir sus derechos, que conocerá sus obligaciones y que tendrá la disposición para colaborar en el rescate de su comunidad.

La apuesta de este trabajo de evaluación y análisis de las noticias bajo la perspectiva de la cultura de la legalidad, es que los medios se involucren de forma activa en la reconstrucción del tejido social de México, es un momento crítico en el que todos los actores e instituciones deben participar y aportar desde su trinchera. Los medios de comunicación tienen el poder de difundir y la forma de llegar a grandes públicos, la intención es que poco a poco, como se ha logrado en otros países, los noticieros y periódicos se vuelvan aliados de los lectores y las audiencias. 
Sí, el problema de la inseguridad y de la violencia en nuestro país tiene que ver en gran medida con la ineficiencia de las autoridades y las instituciones, es notable que no hay una impartición de justicia clara ni eficiente, pero también es necesario considerar que la otra parte de responsabilidad recae en los ciudadanos. El principal problema de la sociedad mexicana ante la ley es una cuestión de actitud y comprensión sobre la naturaleza de lo legal, como lo explica Cortés (2006), "pareciera prevalecer, en ciertos sectores, una visión donde la ley no se concibe como lo que es en una democracia: la auto-imposición de normas públicas por parte de una comunidad de ciudadanos libres. Más bien, la ley suele ser vista todavía como si fuera una imposición externa..." (p. 41). En la medida en que todos participen en exigir a las autoridades y estén dispuestos a colaborar con ella, existen más posibilidades de que se logre establecer un estado de derecho: cultura de la legalidad mejor que cultura de la violencia.

\section{Referencias}

(2006) CORTÉS, Marco Antonio, Cultura de la legalidad en México: creencias sobre la justicia, la ley las Instituciones. Acta republicana. Política y Sociedad, 5, ISSN 1665-9953, México pp. 41-51.

(2010) GARZÓN, Juan Carlos. Narcotraficantes, carteles y otros demonios: violencia e ilegalidad en México y Colombia. Serie Policy Briefs No.10. Publicación electrónica del Consorcio Global para la Transformación de la Seguridad, Chile. <http://www.securitytransformation.org/esp/gc_publications>

(2000) GODSON, Roy, Guide to developing a culture of lawfulness. Trends in Organized Crime, 5, ISSN 1936-4830, pp. 91-110.

Índice Nacional de Corrupción y Buen Gobierno. Informe Ejecutivo 2010. Mayo de 2011. Publicación electrónica de Transparencia Mexicana, México. <http://www.transparenciamexicana.org.mx/ENCBG/>

(2010) Informe sobre seguridad ciudadana y derechos humanos. Resumen. Comisión Interamericana de Derechos Humanos y Organización de los Estados Americanos, México, ISBN 978-0-8270-5431-8.

(2006) LAVEAGA, Gerardo, La cultura de la legalidad, Instituto de Investigaciones Jurídicas UNAM, México.

National Strategy Information Center. Developing support for the Rule of Law: A cultura of Lawlfulness. Página electrónica

<http://www.strategycenter.org/programs/education-for-the-rule-of-law/>

Observatorio de Medios y Cultura de la Legalidad. Página electrónica <http://www.mediosyculturalegal.com>

(2003) ORLANDO, Leoluca, Hacia una cultura de la legalidad. La experiencia Siciliana. Pontificia Universidad Católica de Perú, Perú.

(2006) SALAZAR, Pedro, Democracia y (cultura de la) Legalidad, Instituto Federal Electoral, México. 